\title{
INFLUÊNCIA DE VERMICOMPOSTO NA PRODUÇÃO DE MUDAS DE PINUS ELLIOTTII ENGELM
}

\author{
Influence of vermicompost in the production of Pinus elliottii \\ Engelm. seedlings
}

\author{
Marcos Vinicius Winckler Caldeira \\ Mauro Valdir Schumacher" \\ Elda Raquel Vargas de Oliveira \\ Edson Luís Piroli Luciano \\ Farinha Watzlawick
}

\section{Resumo}

O presente estudo foi conduzido em uma casa de vegetação do Centro Tecnológico de Silvicultura do Departamento de Ciências Florestais, da Universidade Federal de Santa Maria, no Rio Grande do Sul, com a espécie Pinus elliottii Engelm, no período de janeiro a abril de 1997. O presente trabalho teve com objetivo avaliar o efeito de diferentes doses de vermicomposto $\left(0 ; 5,0 ; 10,0 ; 15,0\right.$ e 20,0 $\left.\mathrm{cm}^{3}\right)$ em tubetes com capacidade de receber aproximadamente $50 \mathrm{~cm}^{3}$ de substrato a base de solo (horizonte A $(0-20 \mathrm{~cm})$ da Unidade de Mapeamento São Pedro - Podzólico Vermelho Amarelo). Para correção do pH, isto é, elevar o pH a 6,0 utilizaram-se $\mathrm{CaCO}_{3}$ e $\mathrm{MgCO}_{3}$ na proporção de 2:1 (300g:150g). Constatou-se após 100 dias de desenvolvimento que as mudas responderam de maneira distinta às doses de vermicomposto e recomendam-se doses entre 10,0 (20\%) e 20,0 (40\%) $\mathrm{cm}^{3}$ vermicomposto para produzir mudas com bom padrão de qualidade.

Palavras-chave: Produção de mudas, Pinus elliottii, Nutrição, Vermicomposto, Substrato.

\section{Abstract}

The current study was conducted in a vegetable house of the Silviculture Technological Center of the Forest Science Department, at Santa Maria Federal University, in Rio Grande do Sul with Pinus elliottii from January to April of 1997. The objective of this work was evaluate the effect of different doses of vermicompost ( $0 ; 5.0$; $10.0 ; 15.0$ and $\left.20.0 \mathrm{~cm}^{3}\right)$ in containers with capacity about $50 \mathrm{~cm}^{3}$ of soil substratum (horizon A $(0-20 \mathrm{~cm})$ of São Pedro Unit mapped as - yellow red Podzolic). $\mathrm{CaCO}_{3}$ and $\mathrm{MgCO}_{3}$, with the proportion of 2:1 (300g:150g), was utilized for the $\mathrm{pH}$ correction until to achieve the level 6.0. After 100 days of growth development, the same seedlings presented different results in relation to the vermicompost doses. It is recommended $10(20 \%)$ and $20(40 \%) \mathrm{cm}^{3}$ of vermicompost for the seedling production to reach a good quality pattern.

Keywords: Seedling production, Pinus elliottii, Nutrition, Vermicompost, Substratum.

\footnotetext{
Eng. ${ }^{\circ}$ Florestal, Doutorando em Ciências Florestais, Centro de Ciência Florestais e da Madeira/UFPR. Bolsita do CNPq. Av. Pref. Lothário Meissner, 3400, Jardim Botânico, Curitiba - PR, CEP 80120-170 - E-mail: caldeira@floresta.ufpr.br.

"* Professor Dr. do Departamento de Ciências Florestais/CCR/UFSM. Bolsista do CNPq. schuma@ccr.ufsm.br

*** Acadêmica do curso de Engenharia Florestal, CCR/UFSM

Eno* ${ }^{\circ} \quad$ Florestal, M. Sc. em Engenharia Agrícola/CCR/UFSM

*aten Doutorando em Ciências Florestais, Prof. do Centro de Ciências Florestais e da Madeira/UFPR
} 


\section{Introdução}

O Pinus sp foi introduzido no Brasil no fim do século passado. Originário da América do Norte, o gênero Pinus encontrou em nosso meio as condições ideais para um notável crescimento, principalmente em altitudes superiores a $700 \mathrm{~m}$ s.n.m. Plantado inicialmente em pequena escala, após algumas décadas, passou a ocupar extensas áreas em função do tipo de demanda industrial.

Ao lado da expansão da cultura do Pinus no Brasil, começaram a surgir críticas ao gênero, apoiadas em algumas verdades e muitas lendas. As principais críticas se relacionavam tanto a aspectos gerais como a específicos. Nos primeiros se inclui o fato de ser o Pinus uma espécie exótica, como se isso fosse exclusividade do gênero, quando há outras, também exóticas, ligadas às atividades agrícolas do país. Por outro lado, são destacados os possíveis prejuízos e perigos da monocultura. As críticas específicas se relacionam a aspectos de clima, solo, flora e fauna.

A produção de mudas florestais, em quantidade e qualidade, é uma das fases mais importantes para o estabelecimento de povoamentos florestais, com grande repercussão sobre a produtividade. Muitos trabalhos têm sido envidados no sentido de melhorar a qualidade e reduzir os custos de produção de mudas (GONÇALVES; POGGIANI, 1996).

A boa formação de mudas destinadas à implantação de povoamentos florestais para a produção de madeira e povoamentos mistos para fins de preservação ambiental e ou recuperação de áreas degradadas, conforme Gonçalves e Poggiani (1996), está relacionada com o nível de eficiência dos substratos, pois a germinação de sementes, iniciação radicial e enraizamento de estacas, formação do sistema radicial e parte aérea está associado com a boa capacidade de aeração, drenagem, retenção de água e disponibilidade balanceada nos mesmos. Essas características são altamente correlacionadas entre si. As duas primeiras estão diretamente relacionadas com a macroporosidade e a retenção de água e nutrientes com a microporosidade e superfície específica do substrato.

De acordo com os autores acima, a presença de um ou mais componentes numa mistura de substrato com partículas de diâmetro menor ou igual ao diâmetro médio dos macroporos da mistura leva ao bloqueio de grande parte da macro- porosidade. Esta é uma situação comum em misturas com predominância de compostos orgânicos, mas que recebem grandes quantidades de terra de subsolo, rico em areia fina e ou muito fina e argila.

O presente trabalho foi desenvolvido com o objetivo de quantificar a dosagem mais adequada de vermicomposto na produção de mudas de Pinus elliottii.

\section{Material e Métodos}

O experimento foi instalado em casa de vegetação climatizada, localizada no Centro Tecnológico de Silvicultura (CTS), pertencente ao Departamento de Ciências Florestais da Universidade Federal de Santa Maria, no período de 17 de janeiro a 27 de abril de 1997.

O clima de Santa Maria, segundo a classificação de Köeppen, é subtropical do tipo Cfa, que se caracteriza por apresentar chuvas durante todos os meses do ano e possuir a temperatura média do mês mais quente superior a $22^{\circ} \mathrm{C}$, e a do mês mais frio superior a $3^{\circ} \mathrm{C}$. A precipitação média varia de 1404 a $1760 \mathrm{~mm}$ (MORENO, 1961).

As sementes utilizadas de Pinus elliottii foram fornecidas pela Fundação Estadual de Pesquisa Agropecuária/Centro de Pesquisa de Florestas e Conservação do Solo, Santa Maria, RS.

As mudas foram produzidas em tubetes de plásticos (polipropileno) com as seguintes dimensões: externa $32 \mathrm{~mm}$; interna $26 \mathrm{~mm}$ e altura $126 \mathrm{~mm}$. O tubete tem uma capacidade de receber aproximadamente $50 \mathrm{~cm}^{3}$ de substrato.

A semeadura foi manual direta, sendo semeadas cinco (5) sementes em cada tubete. Após 7 dias da semeadura foram retiradas as mudas em excesso, deixando uma muda por tubete.

O substrato utilizado para produção das mudas foi o solo coletado no horizonte A (0-20 $\mathrm{cm}$ ) da Unidade de Mapeamento São Pedro (Podzólico Vermelho-Amarelo) (Tabela 1) acrescido de diferentes doses de vermicomposto. Análise química do solo foi realizada no Laboratório de Análises do Solo do Departamento de Solos da Universidade Federal de Santa Maria, conforme a metodologia de Tedesco et al. (1995). Para correção do pH, isto é, elevar o pH a 6,0, utilizaram-se $\mathrm{CaCO}_{3}$ e $\mathrm{MgCO}_{3}$ na proporção de 2:1 (300:150g). 
TABELA 1 - Análise química do solo utilizada na mistura com vermicomposto para produção de mudas de Pinus elliottii

Table 1 - Chemical analysis of the soil used in the mixture with vermicompound for seedling production of Pinus elliottii

\begin{tabular}{ccccccccccc}
\hline $\begin{array}{l}\mathrm{pH} \\
\mathrm{H}_{2} \mathrm{O}\end{array}$ & $\mathrm{P}$ & $\mathrm{K}$ & $\mathrm{MO}$ & $\mathrm{Al}$ & $\mathrm{Ca}$ & $\mathrm{Mg}$ & $\mathrm{H}+\mathrm{Al}$ & $\begin{array}{r}\mathrm{CTC} \\
\text { efetiva }\end{array}$ & $\begin{array}{c}\mathrm{CTC} \mathrm{pH} \\
\text { a } 7,0\end{array}$ & $\begin{array}{c}\mathrm{V} \\
(\%)\end{array}$ \\
\hline \multicolumn{1}{c}{$\mathrm{mg} / \mathrm{l}$} & $\mathrm{g} / \mathrm{kg}$ & \multicolumn{7}{c}{$\mathrm{Cmol}_{\mathrm{c}} / \mathrm{l}$} \\
\hline 4,9 & 5,5 & 42,0 & 21 & 1,5 & 2,9 & 3,0 & 10,5 & 7,5 & 16,5 & 36,0 \\
\hline
\end{tabular}

O vermicomposto utilizado no trabalho foi proveniente de produção de minhocas vermelhas da Califórnia (Eisenia foetida Savigny, 1826) a partir de esterco de bovino. Pode-se observar na Tabela 2 análise química do vermicomposto.

A análise química do vermicomposto foi realizada no Laboratório de Análise de Solos do Departamento de Solos da Universidade Federal de Santa Maria, segundo a metodologia proposta por Tedesco et al. (1995).

TABELA 2 - Análise química do vermicomposto utilizado na produção de mudas de Pinus elliottii Table 2 - Chemical analysis of the vermicompound used in the seedling production of Pinus elliottii

\begin{tabular}{|c|c|c|c|c|c|c|}
\hline \multicolumn{7}{|c|}{ Elementos Analisados } \\
\hline $\mathrm{PH}$ & $\mathrm{Da}^{*}$ & N Total & P Total & K Total & & a Total \\
\hline & & \multicolumn{2}{|c|}{$(g / k g)$} & \multicolumn{3}{|c|}{$(g / k g)$} \\
\hline 7,0 & 0,12 & 1,1 & 2,6 & 6,0 & & 5,4 \\
\hline \multicolumn{7}{|c|}{ Elementos Analisados } \\
\hline Mg Total & Fe Total & S Total & B Total & Mn Total & Cu Total & Fe Total \\
\hline \multicolumn{3}{|c|}{$(\mathrm{mg} / \mathrm{kg})$} & \multicolumn{4}{|c|}{$\overline{(\mathrm{mg} / \mathrm{kg})}$} \\
\hline 3,4 & 2,0 & 2,5 & 27 & 824 & 20 & 2,0 \\
\hline
\end{tabular}

* Densidade aparente

O delineamento experimental foi inteiramente casualizado, com cinco tratamentos (Tabela 3) e cinco repetições, com 50 plantas por tratamento. Para diâmetro e altura foram medidas todas as planta. Entretanto, para quantificar massa seca biomassa aérea e radicial foram utilizadas 40 plantas aleatoriamente por tratamento.

TABELA 3 - Doses de vermicomposto utilizadas na produção de mudas de Pinus elliottii Table 3 - Doses of vermicompound used in the seedling production of Pinus elliottii

\begin{tabular}{cc}
\hline Trat. & Dose de vermicomposto em $\left(\mathrm{cm}^{3}\right)$ \\
\hline T1 & 0 (sem vermicomposto - somente solo) do volume total do tubete $=50 \mathrm{~cm}^{3}$ \\
T2 & $10 \%$ ou $5,0 \mathrm{~cm}^{3}$ (do volume total do tubete $\left.=50 \mathrm{~cm}^{3}\right)$ \\
T3 & $20 \%$ ou $10,0 \mathrm{~cm}^{3}$ (do volume total do tubete $\left.=50 \mathrm{~cm}^{3}\right)$ \\
T4 & $30 \%$ ou $15,0 \mathrm{~cm}^{3}$ (do volume total do tubete $\left.=50 \mathrm{~cm}^{3}\right)$ \\
T5 & $40 \%$ ou $20,0 \mathrm{~cm}^{3}$ (do volume total do tubete $\left.=50 \mathrm{~cm}^{3}\right)$ \\
\hline
\end{tabular}


Após 100 dias da semeadura, as variáveis analisadas foram: diâmetro do colo, altura total de planta, massa seca aérea e radicial.

Para determinação da massa seca, as raízes foram separadas da parte aérea com um auxílio de uma tesoura. As mesmas foram lavadas utilizando-se peneira de $0,75 \mathrm{~mm}$ e $0,210 \mathrm{~mm}$ de malha. A parte aérea e radicial separadamente foram colocadas em sacos de papel pardo para secar em estufa com temperatura de $75^{\circ} \mathrm{C}$, até atingirem peso constante, o que foi feito por amostragem diária, isto é, duas vezes por dia aleatoriamente pesaram-se alguns tratamentos para verificar se os mesmos atingiram o peso constante. Peso de matéria seca total foi obtido somando-se as duas partes.

Obteve-se a média por planta para cada variável medida, e com esses valore analisou-se estatisticamente, pelo programa SPSS (Statistical Package for the Social Science) versão 7.5 para Windows, comparando os resultados de cada tratamento.
Para a determinação da curva de resposta de crescimento das mudas de Pinus elliottii, em função das diferentes doses de vermicomposto, foram testados vários modelos de regressão. O melhor modelo foi $\mathbf{y}=\mathbf{b}_{0}+\mathbf{b}_{\mathbf{1}} * \mathbf{x}_{\mathbf{1}}+$ $\mathbf{b}_{2} * \mathbf{x}_{2}{ }^{2}$, onde $\mathrm{x}=$ doses de vermicomposto e $\mathrm{y}=$ variáveis de interesse (diâmetro do colo, altura total de planta, massa seca aérea, radicial e total).

\section{Resultados e Discussão}

A Tabela 4 mostra os valores médios das variáveis analisadas em mudas de Pinus elliottii em função de diferentes doses de vermicomposto. Para as variáveis altura, diâmetro do colo e massa seca radicial mudas produzidas com 5,0; 10,0; 15,0 e $20,0 \mathrm{~cm}^{3}$ de vermicomposto não diferiram estatisticamente.

TABELA 4-Valores médios de altura (H), diâmetro do colo (D), massa seca aérea (MSA), de raízes (MSR) e total (MST) em função de diferentes doses de vermicomposto, aos 100 dias após a semeadura de Pinus elliottii

Table 4-Average values of height (H), diameter of the base (D), aerial dry mass (MSA), dry mass of roots (MSR) and total (MST) in function of different doses of vermicompound, to the 100 days after the sowing of Pinus elliottii

\begin{tabular}{cccccc}
\hline Trat. & $\mathrm{H}$ & $\mathrm{D}$ & MSA & MSR & MST \\
\cline { 2 - 6 } & $(\mathrm{cm})$ & $(\mathrm{mm})$ & & g/planta & \\
\hline T1 & $13,2 \mathrm{a}$ & $1,81 \mathrm{a}$ & $0,300 \mathrm{a}$ & $0,192 \mathrm{a}$ & $0,492 \mathrm{a}$ \\
T2 & $16,2 \mathrm{~b}$ & $2,30 \mathrm{~b}$ & $0,505 \mathrm{~b}$ & $0,656 \mathrm{~b}$ & $1,201 \mathrm{~b}$ \\
T3 & $16,8 \mathrm{~b}$ & $2,43 \mathrm{~b}$ & $0,619 \mathrm{c}$ & $0,787 \mathrm{~b}$ & $1,407 \mathrm{bc}$ \\
T4 & $16,0 \mathrm{~b}$ & $2,32 \mathrm{~b}$ & $0,688 \mathrm{c}$ & $0,793 \mathrm{~b}$ & $1,481 \mathrm{c}$ \\
T5 & $15,4 \mathrm{~b}$ & $2,50 \mathrm{~b}$ & $0,663 \mathrm{c}$ & $0,684 \mathrm{~b}$ & $1,397 \mathrm{bc}$ \\
\hline
\end{tabular}

Médias ligadas na vertical com a mesma letra não diferem estatisticamente pelo teste de Tukey em 5\% de probabilidade.

No que se refere à produção de massa seca observa-se, pela Tabela 4 que mudas produzidas com 10,$0 ; 15,0$ e 20,0 $\mathrm{cm}^{3}$ de vermicomposto foram as que obtiveram maior produção de massa seca, não havendo diferença estatística entre esses tratamentos. No entanto, a maior produção de massa seca total foi no tratamento com $15,0 \mathrm{~cm}^{3}$ de vermicomposto, sendo que as mudas dos tratamentos 10,0 e $20,0 \mathrm{~cm}^{3}$ de vermicomposto foram equivalentes.

A Tabela 5 mostra o modelo de regressão ajustado para as variáveis analisadas em mudas de Pinus elliottii produzidas em diferentes doses de vermicomposto. 
TABELA 5 - Equação de regressão ajustada $(\mathrm{Y}=\mathrm{b} 0+\mathrm{b} 1 * \mathrm{X} 1+\mathrm{b} 2 * \mathrm{X} 2)$ para altura $(\mathrm{H})$, diâmetro do colo (D), massa seca aérea (MSA), raízes (MSR) e total (MST) em mudas de Pinus elliottii, em resposta à aplicação de diferentes doses de vermicomposto

Table 5 - Adjusted regression $(Y=b 0+b 1 * X 1+b 2 * X 2)$ for beight $(H)$, diameter of the base (D), aerial dry mass (MSA), dry mass of roots (MSR) and total (MST) in seedlings of Pinus elliottii, as response to the application of different doses of vermicompound

\begin{tabular}{ccc}
\hline Variável & Equação de Regressão & $\mathrm{R}^{2} \mathrm{a}^{*}$ \\
\hline $\mathrm{H}$ & $\mathrm{Y}=13,450514+0,552674 * \mathrm{X}_{1}+(-0,022046) * \mathrm{X}_{2}{ }^{2}$ & 0,33 \\
$\mathrm{D}$ & $\mathrm{Y}=1,869771+0,075371 * \mathrm{X}_{1}+(-0,002148) * \mathrm{X}_{2}{ }^{2}$ & 0,35 \\
MSA & $\mathrm{Y}=0,300884+0,047159 * \mathrm{X}_{1}+(-0,001449) * \mathrm{X}_{2}{ }^{2}$ & 0,58 \\
MSR & $\mathrm{Y}=0,227201+0,096495 * \mathrm{X}_{1}+(-0,003745) * \mathrm{X}_{2}{ }^{2}$ & 0,56 \\
MST & $\mathrm{Y}=0,532371+0,139940 * \mathrm{X}_{1}+(-0,004907) * \mathrm{X}_{2}{ }^{2}$ & 0,68 \\
\hline
\end{tabular}

As mudas de Pinus elliotii responderam de modo significativo às doses de vermicomposto. Observa-se na Figura 1A e B que ocorre uma diminuição no crescimento em diâmetro e altura à medida que aumentam às doses de vermicomposto. Essa observação também foi verificada em $A c a$ cia mearnsii utilizando o mesmo tipo de vermicomposto (CALDEIRA et al., 2000a).

O parâmetro diâmetro de colo, em geral, é o mais observado para indicar a capacidade de sobrevivência da muda no campo. Portanto, é o mais utilizado para auxiliar na definição das doses de fertilizantes a serem aplicadas na produção de mudas (DANIEL et al., 1997).

FIGURA 1 - Crescimento em altura, diâmetro e produção de massa seca aérea, radicial e total de Pinus elliottii, em função de diferentes doses de vermicomposto

Figure 1 - Growth in beight, diameter and production of aerial, rootstock and total dry mass of Pinus elliottii, in function of different doses of vermicompound

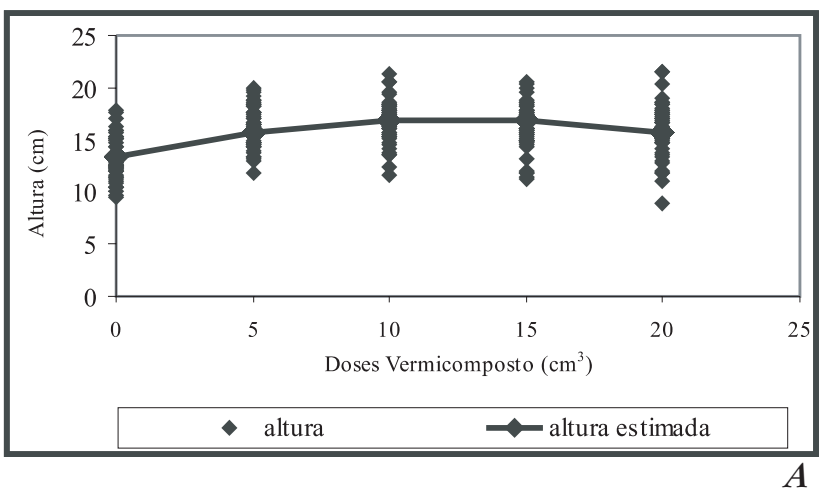

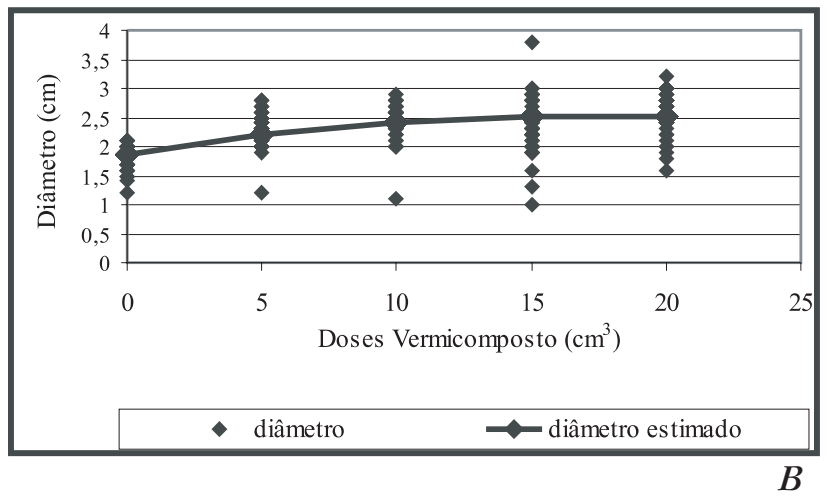
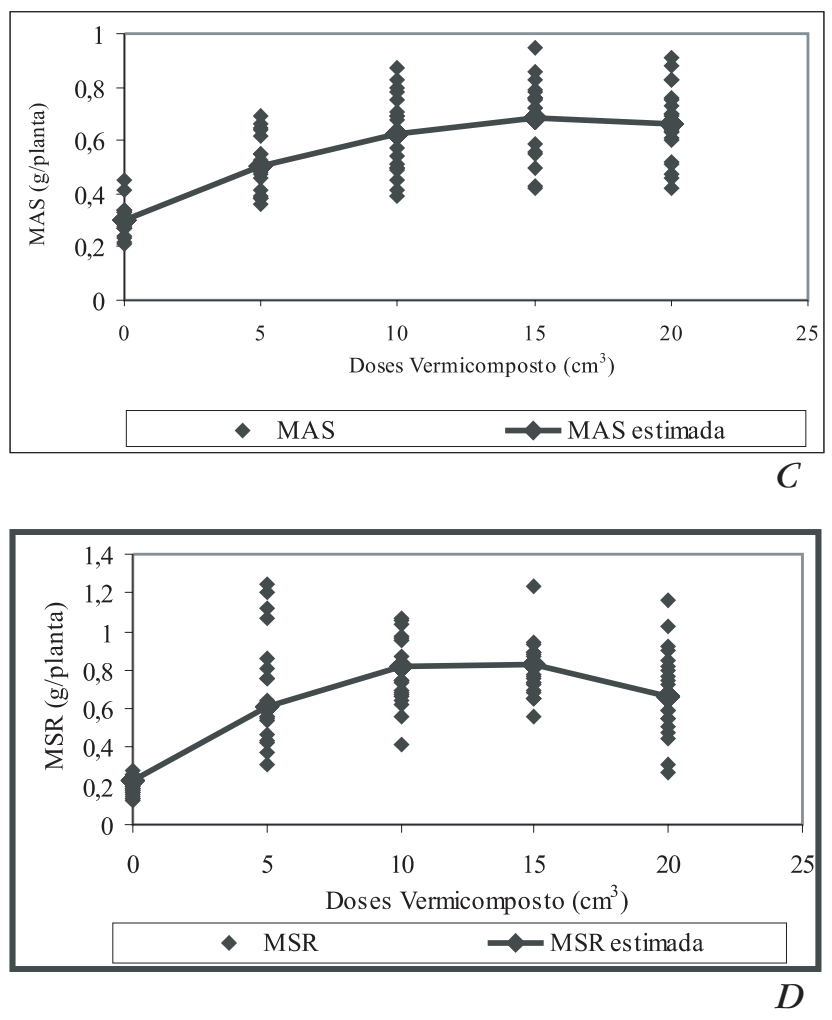


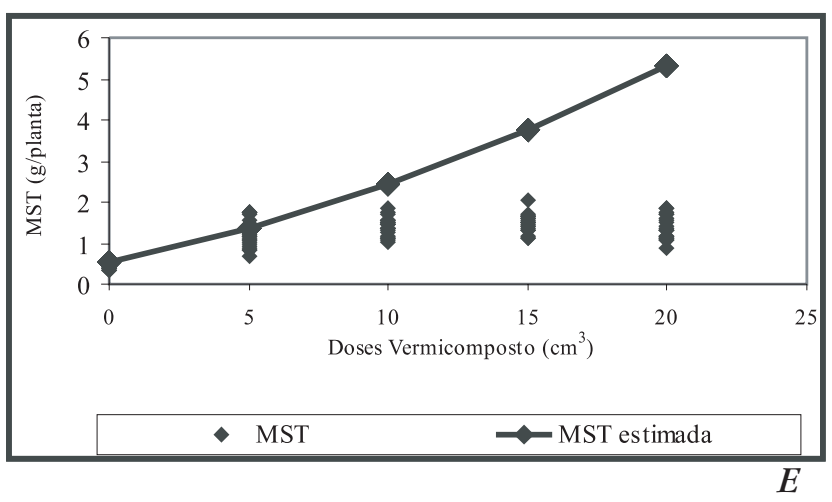

A dose de vermicomposto a ser usada deve ser escolhida em função da necessidade na produção das mudas, pois se a intenção é produzir mudas de Pinus elliottii com boa altura e/ou diâmetro de colo, por exemplo, não há necessidade de aplicar mais que $10 \mathrm{~cm}^{3}$ de vermicomposto (Figura 1A, B). (ALVES; PASSONI, 1997; TEDESCO et al., 1999).

Para a variável massa seca aérea e radicial observa-se na Figura 1C e D que à medida que aumentam as doses de vermicomposto ocorre uma diminuição na produção de ambas massas secas com exceção da massa seca total (Figura E). Estudo realizado por Caldeira et al. (2000a) em Acacia mearnsii verificaram que ocorre uma diminuição na massa seca aérea e radicial com o aumento das doses de vermicomposto. A observação da Figura 1C e D significa que, provavelmente, mudas de Pinus elliottii não suportam doses elevadas de vermicomposto na produção de mudas. Mesmo assim, durante os 100 dias de desenvolvimento não foram constatados efeitos fitotóxicos.

A utilização de composto orgânico e vermicomposto como substrato para produção de mudas é em função da exigência das espécies, pois estudo realizado por Alves e Passoni (1997) teve como objetivo avaliar composto orgânico e vermicomposto oriundos do lixo domiciliar, como substrato para produção de oiti (Licania tomentosa). Os autores observaram que mesmo nas doses mais elevadas de composto orgânico ou vermicomposto, até mesmo na substituição total do solo por estes adubos, não ocorreram problemas de germinação ou desenvolvimento das mudas; havendo, ao contrário, melhoria no desenvolvimento, em comparação com a testemunha. Isso sugere a não ocorrência de efeito fitotóxico, atribuível a possíveis contaminações existentes no composto orgânico ou vermicomposto. Tal fato pode ser explicado conforme Garcia apud Hernàndez et al. (1992) pela estabilização ou eliminação de substâncias fitotóxicas, promovidas pelo processo de compostagem. Pode-se observar na Figura 2 que a menor Razão foi no tratamento em que as mudas foram produzidas sem a utilização de vermicomposto. Segundo Glass citado por Daniel et al. (1997), a razão aumenta à medida que diminui o suprimento de nutrientes, pois a sua diminuição está relacionada com uma melhor nutrição no substrato.

FIGURA 2 - Valores da Razão MSR/MAS de Pinus elliottii, em função de diferentes doses de vermicomposto

Figure 2 - Values of the ratio MSR/MAS for Pinus elliottii, in function of different doses of vermicompound

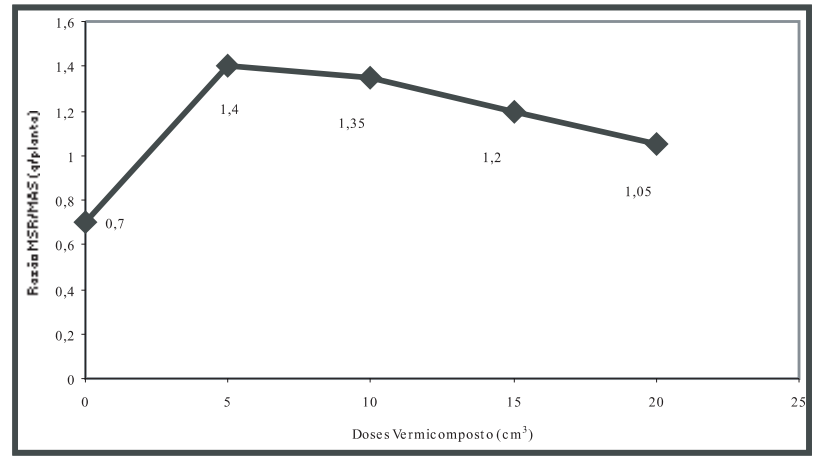

Não se pode constatar a estabilização da razão em 1,05 com $20 \mathrm{~cm}^{3}$ de vermicomposto. A tendência, pela observação, é continuar diminuindo na medida em que vai aumentando as doses de vermicomposto (Figura 2). Resultados contrários foram observados por Caldeira et al. (2000a) utilizando o mesmo tipo de vermicomposto em mudas de Acacia mearnsii . Porém, Caldeira et al. (2000b) e Barichello et al. (2001) observaram que não houve estabilização da razão em mudas de $E$. saligna e camaldulensis, respectivamente. Tedesco et al. (1999) verificaram em mudas de Jacaranda micrantha (1999) que as doses de vermicomposto tendem a diminuir a razão. Neste sentido, a razão é em função da espécie, do tipo do substrato a ser utilizado na produção de mudas, bem como da fertilidade do mesmo (CALDEIRA et al., 2000a).

A razão é comumente maior em ambiente de baixa fertilidade, podendo ser uma estratégia da planta para retirar o máximo de nutrientes naquela condição (CLARKSON, 1985). 


\section{Considerações Finais}

Em todos os parâmetros analisados, doses de vermicomposto influenciam no crescimento de mudas de Pinus elliottii.

Não há sintomas de fitotoxicidade nas mudas de Pinus elliottii atribuídas à utilização de vermicomposto.

Para produzir mudas de Pinus elliottii com um bom padrão de qualidade deve-se utilizar doses de vermicomposto entre $10-20 \mathrm{~cm}^{3}$ por tubete de $50 \mathrm{~cm}^{3}$.

Doses crescentes de vermicomposto tendem a diminuir o crescimento de mudas de Pinus elliottii.

\section{Referências}

AlveS, W. L.; PASSONI, A. A. Composto e vermicomposto de lixo urbano na produção de mudas de oiti (Licania tomentosa (Benth.) para arborização. Pesquisa Agropecuária Brasileira, Brasília, v. 32, n. 10, p. 1053-1058, 1997.

BARICHELLO, L. R. et al. Utilização de vermicomposto no crescimento de mudas de Eucalyptus camaldulensis Dehnh. Revista Árvore, Viçosa, v. 25, n .4, p. 397-402, 2001.

CALDEIRA, M. V. W. et al. Crescimento de mudas de Eucalyptus grandis W. Hill ex Maiden em função de diferentes doses de vermicomposto. Revista Floresta, Curitiba, v. 28, n. 1/2, p. 19-30, 2000b.

CALDEIRA, M. V. W.; SCHUMACHER, M. V.; TEDESCO. N. Crescimento de mudas de Acacia mearnsii em função de diferentes doses de vermicomposto. Scientia Forestalis, Piracicaba, n. 57, p. 161-170, 2000a.
CLARKSON, D. T. Adaptação morfológica e fisiológica das plantas a ambientes de baixa fertilidade. In: SIMPÓSIO SOBRE RECICLAGEM DE NUTRIENTES E AGRICULTURA DE BAIXOS INSUMOS NOS TRÓPICOS, Ilhéus, 1984. Anais... Ilhéus: CEPLAC/SBSC, 1985. p. 45-75.

DANIEL, O. et al. Aplicação de fósforo em mudas de Acacia mangium WILLD. Revista Árvore, Viçosa, v. 21, n. 2, p. 163-168, 1997.

GONÇALVES, J. L. M.; POGGIANI, F. Substrato para produção de mudas florestais. In: SOLOSUELO- CONGRESSO LATINO AMERICANO DE CIÊNCIA DO SOLO, 13, 1996. Resumos expandidos... Águas de Lindóia-SP, 1996. Águas de Lindóia: SLCS: SBCS: ESALQ/USP: CEA-ESALQ/USP: SBM, 1996. CD-ROM.

HERNÁNDEZ, T. C. et al. Utilization of municipal wastes as organic fertilizers. Suelo y Planta, v. 2, n. 3, p. 373-383, 1992.

moreno, J. A. Clima do Rio Grande do Sul. Porto Alegre: Secretaria da Agricultura, 1961.

TEDesco, J. M. et al. Análise de solo, plantas e outros materiais. 2. ed. rev. e ampli. Porto Alegre: Universidade Federal do Rio Grande do Sul, 1995. 174 p. (Boletim Técnico, n. 5).

TEDESCO, N.; CALDEIRA, M. V. W.; SCHUMACHER, M.V. Influência do vermicomposto na produção de mudas de caroba (Jacaranda micrantha Chamisso). Revista Árvore, Viçosa, v. 23, n. 1, p. 1-8, 1999. 